\title{
Relationship between Average Coordination Number and Void Fraction in Randomly Packed Systems of Uniform-sized Spheres Developed by Four Kinds of Computer Simulation ${ }^{\dagger}$
}

\author{
Michitaka Suzuki and Toshio Oshima \\ Department of Chemical Engineering \\ Himeji Institute of Technology*
}

\begin{abstract}
The relationship between the average coordination number and void fraction in a randomly packed system of uniform-sized spheres was investigated by four different kinds of computer simulated results to judge whether or not the coordination number could be uniquely estimated from the voidage. The relationship between the coordination number and voidage depends on the type of the computer simulation program used and our research results showed clearly that the coordination number cannot be uniquely estimated from the void fraction. These computer simulated results were compared with the calculated results using some empirical or model equations which have already been reported in the literature. The model equations by Gotoh and Suzuki et al. and the empirical equations by Sunada et al. and Nagao were found to be in fairly good agreement with some of our simulated results.
\end{abstract}

\section{Introduction}

The coordination number of a particle system or the number of contacts per particle, is not only a means of expression for particle arrangement structures but also a very important value related to the dynamic, heatconduction and electric characteristics of powder. Empirical formulae or model formulae expressing the relationship between the average coordination number and void fraction in randomly packed systems of uniform-sized spheres have been proposed and put into practical use by $\operatorname{Rumpf}^{1)}$, Meissner et al. ${ }^{2)}$, Ridgeway and Tarbuck ${ }^{3)}$, Haughey and Beveridge ${ }^{4)}$, Nakagaki and Sunada ${ }^{5)}$, Smith et $a l^{6)}$, Gotoh ${ }^{7)}$, $\mathrm{Nagao}^{8)}$, the authors ${ }^{9}$, Ouchiyama and Tanaka ${ }^{10}$ and others. However, the average coordination number obtained from the void fraction using the above formulae

* 2167, Shosha, Himeji-shi, Hyogo, 671-22 TEL. 0792-66-1661

$\dagger$ This report was originally printed in J. Soc. Powder Technology, Japan, 25, 204-208 (1988) in Japanese, before being translated into English with the permission of the editorial committee of the Soc. Powder Technology, Japan. varies according to the formula. This variation, in the case of empirical formulae, appears depending on whether the base experimental values stemming from regular packing, liquid bridging or computer simulation. In the case of model formulae, variation occurs according to the type of model used to obtain the formulae, such as whether it is a regular-packing combination model or random-packing model.

This paper reports on two surveys:

(1) A survey, using results of four different computer simulation algorithms, regarding whether the average coordination number of randomly packed systems of uniformsized spheres can be determined uniquely by the void fraction.

(2) A survey as to the extent to which the above empirical and model formulae ${ }^{1 \sim 10}$ ) are applicable to the computer simulated results.

\section{Computer simulation}

The form randomly-packed systems of uniform-sized spheres, we used four computer simulation programs expressing different packing methods. These programs $a$ to $d$ are out- 
arranged in a dendroid structure in the $Z$ direction in Fig. 1.

\section{1 Program $a$}

Spheres, whose $X-Y$ coordinates have been respectively determined by uniform random numbers, were dropped and packed into a rectangular-parallelepiped container whose base plane is a square, each side of which is 15 times longer than the particle diameter. This, if one had done nothing more, would have resulted in void fraction $\epsilon$ being constant at $0.423^{11)}$. To obtain layers with different void fractions, therefore, we introduced the concept of the interparticle friction angle. That is, we modified the program ${ }^{9}$ in such a way as to allow particles to remain in the same place when the zenithal angle $\phi$ is smaller than interparticle friction angle $\phi_{c}$, and to roll down along the particles when the angle is larger. (Here, the zenithal angle $\phi$ is the angle of the contact point at which a falling particle comes in contact with an already-dropped particle.) This revised program enables the obtaining of particlepacked layers with different void fractions by packing particles for various interparticle friction angles $\phi_{c}$. Figure 1 illustrates a typical $Y$ $Z$ cross section of a particle-packed layer simulated by this revised program (where void fraction $\epsilon=0.7$ and $\phi_{c}=0.73 \mathrm{rad}^{*}$.) The base plane is provided with irregular ups and downs to prevent the effect of base plane on the packing structure. We, therefore, calculated the void fraction and coordination number of particlepacked layers in such a range that is not affected by the base plane and the packed layer surface.

To prevent the sidewalls of the container from affecting the particle layers, we used the periodic boundary conditions with complete continuity at the boundaries, so that, for example, a part of the particles which were protruding out of the right side of the container could emerge on the left side of the container. The maximum void fraction that could result from this program, when $\phi_{c}$ is maximized $(=\pi / 2)$, is 0.841 .

On the cross section, we drew a straight line connecting the centers (i.e., the centers of circles representing the cross sections of particles) of the particles contacting one another to allow the particle packing structure to be easily understood. We can see how the particles are

\section{2 Program $b$}

As in program $a$, we determined the $X-Y$ coordinates of spheres in random numbers and applied particles one by one. Like in the simulation program developed by Nakagaki and Sunada ${ }^{5,12)}$, a uniform random number was set up when a falling particle comes in contact with an already-packed particle. When this uniform random number is smaller than predetermined particle adhesion probability $P_{c}$, a specific particle stays adhered there. And when larger, the particle rolls over the surface of such a packed particle. Particle layers with different void fractions can be obtained by varying interparticle adhesion probability $P_{c}$. Figure 2 shows typical $Y-Z$ cross section of a particle layer

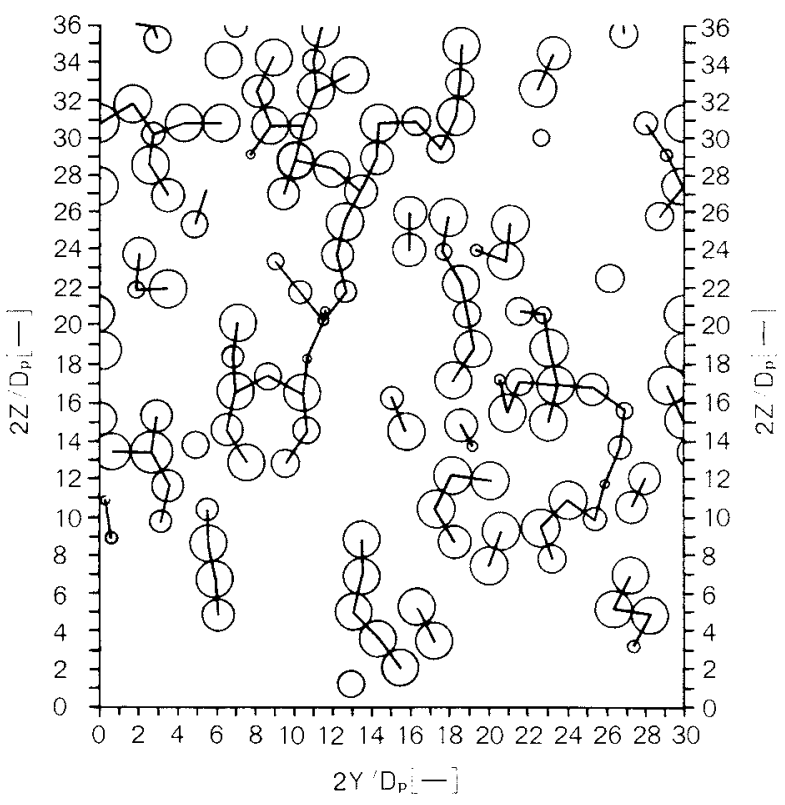

Fig. 1 Cross section of particle system by computer simulation program $a$

* For programs $a$ and $b$, we calculated interparticle friction angle $\phi_{c}$ and particle adhesion probability $P_{C}$ which make void fraction $\epsilon=0.7$, as follows:

(1) changing $\phi_{c}$ and $P_{c}$, several creating particle layer patterns, and calculating value $\epsilon$ of each pattern;

(2) applying the least squares method to the result of step (1) above and calculating an approximate expression indicating the relationships of $\phi_{c}-\epsilon$ and $P_{c}-\epsilon$; and

(3) using an approximate expression to set up $\phi_{c}$ and $P_{c}$ to make $\epsilon=0.7$. 
simulated by this program, where void fraction $\epsilon=0.7$ and $P_{c}=0.4867 *$. Like in program $a$, we set up void fraction and coordination number for a particle layer in such a range that is not affected by the unevenness of the base plane or surface of the layer, and used the periodic boundary conditions to prevent the sidewalls of the container from affecting the layer. The maximum void fraction that can stem from this program, where $P_{c}$ is maximized $(=1)$, is 0.837 .

Like in program $a$ again, the particles were arranged in a dendroid manner. However, the use of adhesion probability results in less orientation in the $Z$ direction than in the case of program $a$, which used interparticle friction angle.

\subsection{Program $c$}

The first step we took on this program was to apply spheres without using interparticle adhesion or friction angle, i.e., under the same condition as in program $a$, where $\phi_{c}=0$ or, as in program $b$, where $P_{c}=0$. This, if one had done nothing more, would have resulted in a void fraction of $0.423^{11)}$. We, therefore, created particle layers with different void fractions by using a uniform random number to selectively remove a specified number of particles from the particles in the particle layer (which are numerized in order of arrival). The particle layer created on this program contains cavities randomly. This layer is thus equal to a version made by applying the model of Shinohara et al. ${ }^{13), 14)}$, which combines regularly-packed portions and cavities, to a randomly-packed layer.

Figure 3 gives a typical $Y-Z$ cross section of a particle layer simulated on this program, where void fraction $\epsilon=0.7$. Here, the onceformed particle layer is deprived randomly of the particles, so that particles are scarcely structured in a dendroid manner or in a certain orientation.

\section{4 Program $d$}

Unlike programs $a, b$ and $c$, this program creates a particle system by determining all the $X, Y$, and $Z$ coordinates of spheres by a uniform random number, instead of dropping spheres with the help of gravity in the $Z$ direction. When particle $\mathrm{B}$ whose $X, Y$, and $Z$ coordinates had been set up by random numbers happened to overlap existing particle $A$, we moved particle $B$ along the straight line connecting particles $\mathrm{A}$ and $\mathrm{B}$ center-to-center to a position where it did not overlap. We, thus, obtained a contact point between particles $\mathrm{A}$ and B. The particle layer simulated on this pro-

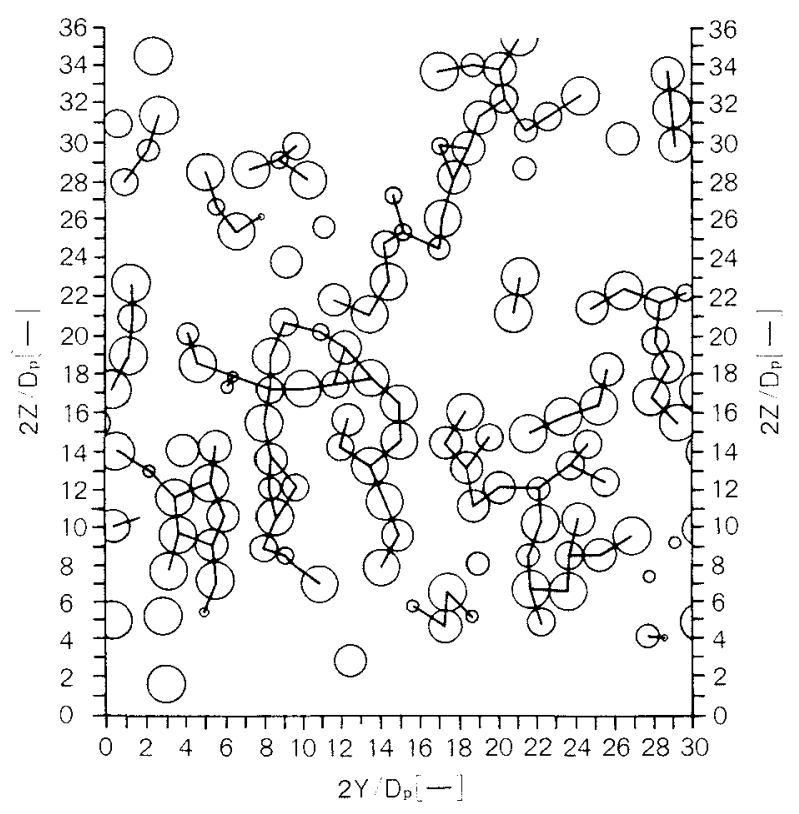

Fig. 2 Cross section of particle system by computer simulation program $b$

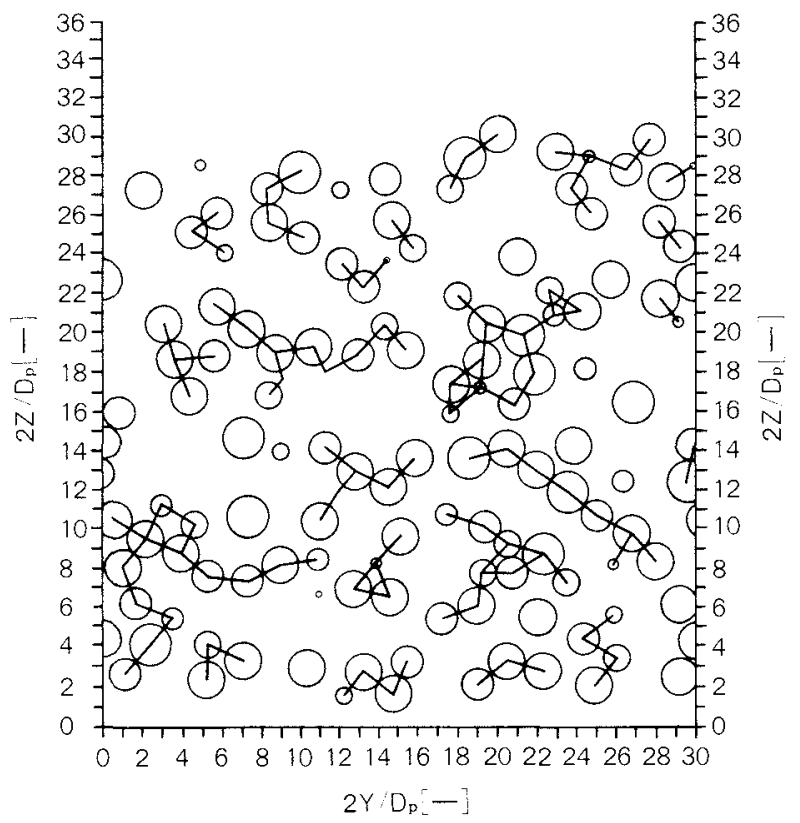

Fig. 3 Cross section of particle system by computer simulation program $c$ 
weram, we believe, shows a particle arrangement pattern similar to partially-coagulated aerosol.

Figure 4 indicates a typical cross section of a particle system created using this method, where void fraction $\epsilon=0.7$. This pattern gave particles randomly in space so that contacts formed only when a particle happened to overlap an existing particle. This pattern, therefore,

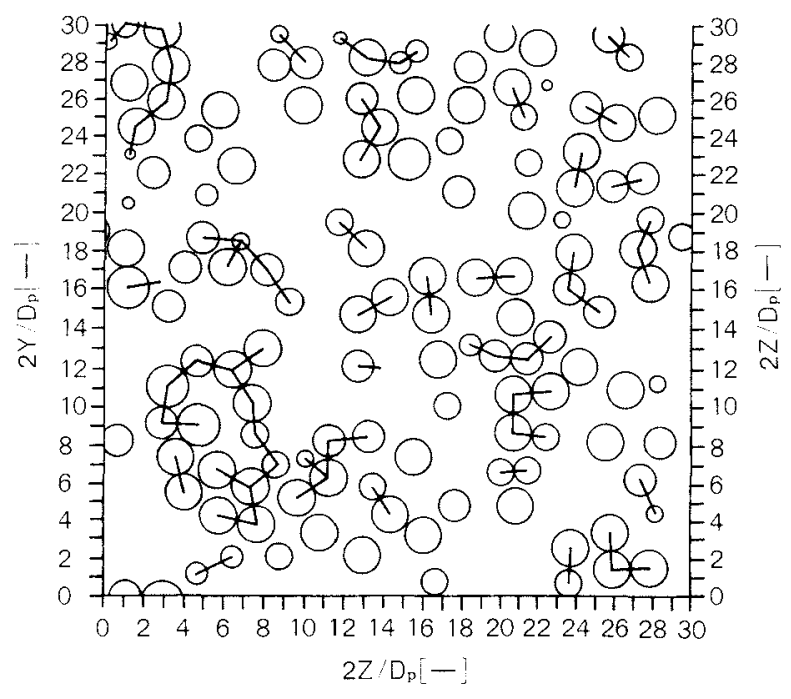

Fig. 4 Cross section of particle system by computer simulation program $d$ shows a smaller number of contacts and less orientation than those given in Figs. $1 \sim 3$.

\section{A comparison of computer simulation with estimation equations}

3. 1 Results of calculations using conventional equations for average coordination number

Table 1 lists the conventional equations showing the relationship between void fraction $\epsilon$ and average coordination number $\bar{N}_{c}$. Of the listed equations, Eqs. (1) (5) proposed by Rumpf ${ }^{1)}$, Meissner et al. ${ }^{2)}$, Ridgeway and Tarbuck $^{3)}$, Haughey and Beveridge ${ }^{4)}$ and $\mathrm{Nagao}^{8)}$ are based on experimental results. Equation (6) by Nakagaki and Sunada ${ }^{5}$ is an empirical formula based on their computer simulated results. Equations (7) and (8) by Smith et al. ${ }^{\text {()) }}$ and Gotoh ${ }^{7)}$ are model formulae stemming from a regular packing pattern. And Eqs. (9) and (10) by the authors ${ }^{9}$ and Ouchiyama and Tanaka ${ }^{10)}$ are model formulae based on a randomly-packed pattern.

Figure 5 compares the results of calculations using the above equations with the results of the aforementioned four kinds of computer simulation. Of the curves representing the calculation results, the broken lines show the results of empirical formulae, while the solid

Table 1 Equations for relation between average coordination number and void fraction in uniform-sized particle system

\begin{tabular}{|c|c|}
\hline Equations & References \\
\hline 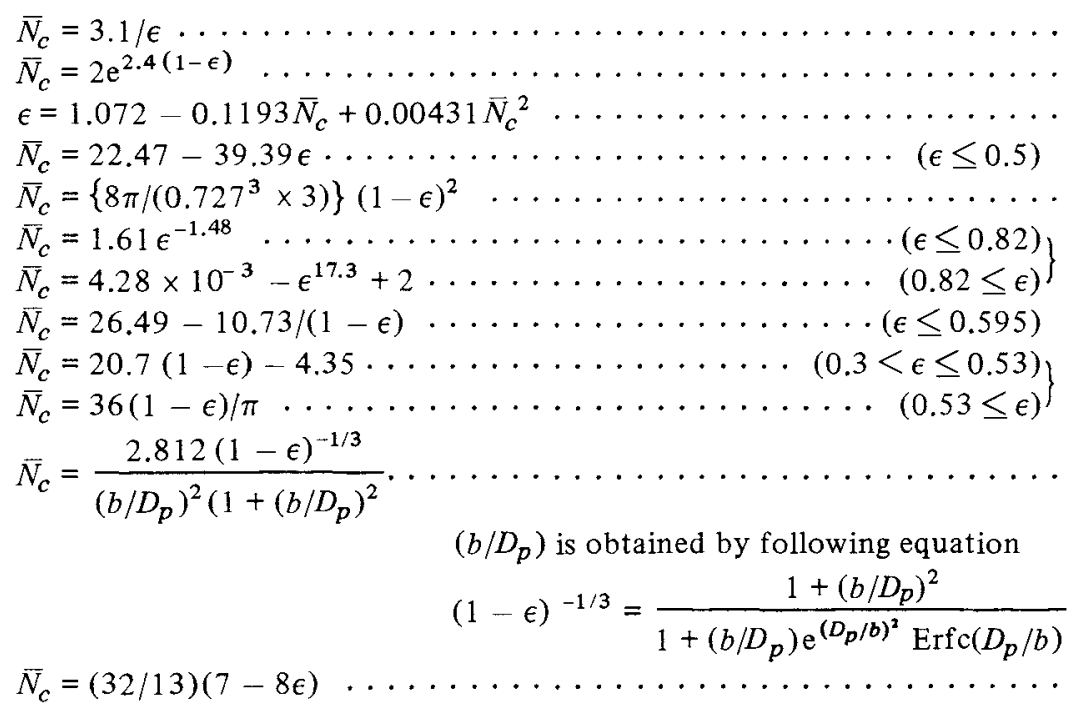 & $\begin{array}{l}\text { (1) Rumpf } \\
\text { (2) Meissner, et al. }{ }^{\text {) }} \\
\text { (3) Ridgeway, et al. } \\
\text { (4) Haughey, et al. } \\
\text { (5) Nagao } \\
\text { 8) } \\
\text { (6) Nakagaki, et al. }{ }^{5)} \\
\text { (7) Smith, et al. }{ }^{6} \\
\text { (8) Gotoh }\end{array}$ \\
\hline
\end{tabular}




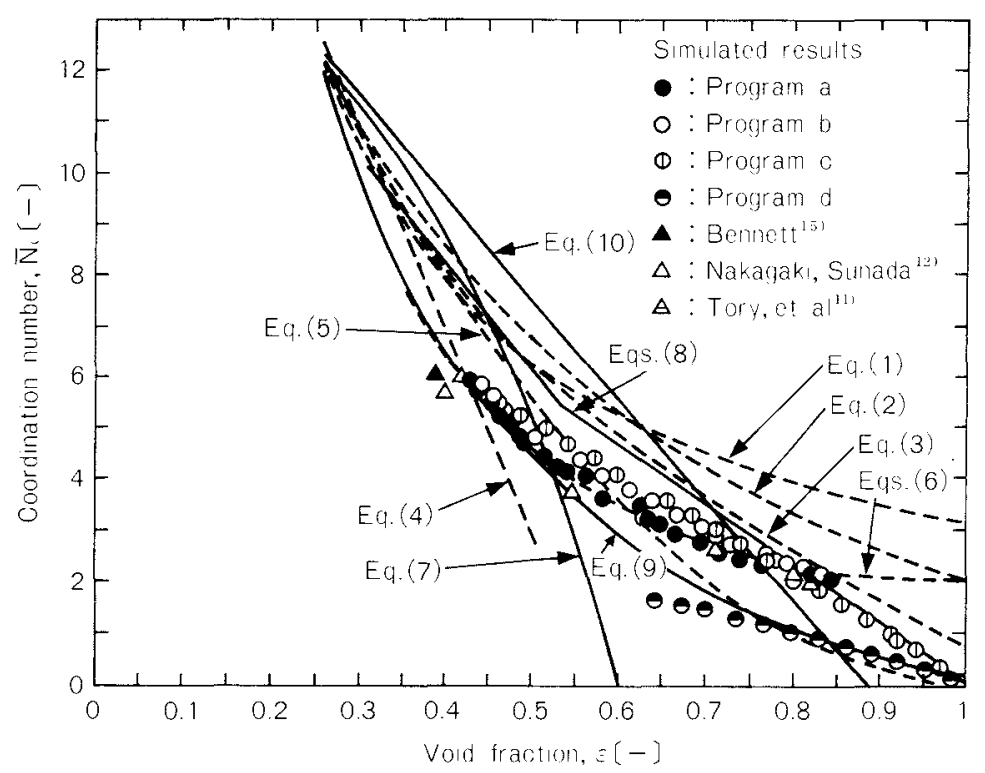

Fig. 5 Comparison of computer simulated results with calculated curves by estimation equations in Table 1

lines are the results of model formulae. As is evident from this figure, conventional estimation equations representing the relationship between void fraction $\epsilon$ and average coordination number $\bar{N}_{c}$ in randomly-packed layer or system of uniform-sized spheres produce different calculation results depending on the type of equation. For example, an estimated average coordination number based on particlelayer void fraction actually measured varies according to the type of equation used. This variation, in the case of empirical formulae, stems from the diversity of methods of creating particle layers and of measuring coordination numbers used in setting up the formulae. In the case of model formulae, this variation is due to the difference in particle layer models depending on the researcher who envisions each model.

\section{2 Results of computer-simulated packing}

Figure 5 illustrates the relationship between the void fraction $\epsilon$ and average coordination number $\bar{N}_{c}$ of particle layers simulated by four different computer programs, which can clearly prescribe particle packing conditions. Here, when void fraction $\epsilon$ ranged from 0.423 to 0.8 , computer simulation on programs $a, b$ and $c$ produced almost the same results, despite some dispersion. However, when $\epsilon$ was equal to or more than 0.8 , programs $a$ and $b$ produced average coordination number $\bar{N}_{c}$ gradually approaching 2 , and program $c$ produced $\bar{N}_{c}$ approaching 0 , as $\epsilon$ approximated 1 . This, we believe, was because of the following:

- On programs $a$ and $b$, particles were arranged in a dendroid pattern despite an increase in interparticle friction angle $\phi_{c}$ and particle adhesion probability $P_{c}$, so that each particle contacted two neighboring particles, one above and the other below:

- On program $c$, particles contacting no neighboring particles increased in number when a once-packed particle layer had been deprived of many particles. Compared with the above programs, program $d$ produces a small average coordination number $\bar{N}_{c}$ at the same void fraction and $\bar{N}_{c}$ gradually approached 0 as $\epsilon$ neared 1 . This $\bar{N}_{c}$ decline with the same $\epsilon$, we presume, is because of the appearance of contacts only when particles whose $X, Y$ and $Z$ coordinates had been determined by random numbers happen to overlap existing particles. In addition to the results of simulation using these four programs, Fig. 5 indicates the results of other major computer simulated results ${ }^{11), 12), 15)}$ reported so far.

3. 3 A comparison of computer simulation results with estimation equations

We compared the results of four kinds of 
weomputer simulation with the results of calculations using equations for average coordination number to find which equation applies best to which packing condition. This reveals that, in the void fraction $\epsilon$ range between 0.42 and 0.8 , the results of calculations using Nakagaki and Sunada's ${ }^{5)}$ empirical Eq. (6) corresponds well to the results of simulation using programs $a, b$, and $c$. This, we believe, is because this empirical formula is based on the above researchers' computer simulation using the concept of interparticle adhesion probability. Even when $\epsilon$ is equal to or more than 0.8 , this formula corresponds well to the results of program $a$ and $b$. To the results of program $c$ with $\epsilon$ equal to or more than 0.8, Gotoh's 7 ) model Eq. (8) corresponds well. To the results of program $d$, on the other hand, Nagao's ${ }^{8)} \mathrm{em}-$ pirical Eq. (5) and the authors'9) model Eq. (9) based on random packing corresponds comparatively well. Equation (9) also corresponded well to the results of simulation using programs $a, b$ and $c$ with $\epsilon$ in the $0.42 \sim 0.55$ range. This, we believe, is because this model formula is based on the homogeneous isotropy of particle arrangement so that it corresponds well to the case when interparticle friction angle and adhesion probability are small and the particles in the particle layer are arranged less directionally and more homogeneously.

Many of the other empirical and model formulae did not correspond well to our simulation results or main conventional simulation results. In fact, the coordination numbers calculated based on these formulae exceeded the comparable simulation results. This, we presume, is because of the following:

- Empirical Eqs. (1) (3): They stem from coordination numbers measured using liquid bridging. As point out by Bernal and Mason ${ }^{16)}$, when coordination numbers are measured by liquid bridging, and it occurs also between particles not in contact, but placed near each other resulting in coordination numbers determined at values exceeding their real values.

- Equations (7): It is based on a model combining two regularly-packed systems so that it fails to correspond to comparable simulation results. Despite being a model formula based on random packing, Eq. (10) fails to correspond to comparable computer simula- tion results.

\section{Conclusion}

Using four different computer programs, we simulated randomly-packed systems of uniformsized spheres to determine their average coordination number and void fraction. This revealed that the relationship between average coordination number and void fraction varied according to the type of program used so that no average coordination number could be determined uniquely by voidage. This led us to conclude that there existed no equation applicable to all circumstances. We then compared simulation results with various empirical and model formulae proposed so far. This comparison showed that Sunada et al.'s empirical formula corresponded well to three, out of the above four, simulation results over a wide void fraction range. Some of the above simulation results corresponded well to the authors' and Gotoh's model formula and Nagao's empirical formula in a certain void fraction range.

\section{Nomenclature}

$$
\begin{array}{rlr}
b & : \text { constant in Eq. (9) } & \\
D_{p} & : \text { particle diameter } & {[\mathrm{m}]} \\
\bar{N}_{c} & : \text { average coordination number } & {[-]} \\
P_{c} & : \text { adhesive probability in } & \\
X, Y, Z & : \text { program } b & {[-]} \\
\epsilon & : \text { voordinates } & {[-]} \\
\phi & : \text { zenithal angle at the first contact } & {[-]} \\
& \quad \text { in program } a & {[\mathrm{rad}]} \\
\phi_{c} & : \text { angle of friction in program } a & {[\mathrm{rad}]} \\
\text { References } & &
\end{array}
$$

\section{References}

1) Rumpf, H.: Chemie Ing. Techn., 30, 144 (1958).

2) Meissner, H.P., A.S. Michaels and R. Kaiser: Ind. Eng. Chem., Process Design and Dev., 3, 202 (1964).

3) Ridgeway, K. and K.J. Tarbuck: Brit. Chem. Eng., 12, 384 (1967).

4) Haughey, D.P. and G.S.G. Beveridge: Can. J. Chem. Eng., 47, 130 (1969).

5) Nakagaki, M. and H. Sunada: Yakugaku Zasshi, 88, 651 (1929).

6) Smith, W. O., P. D. Foote and P. F. Busang: Phys. Rev., 34, 1271 (1929).

7) Gotoh, K.: J. Soc. Powder. Technol., Japan, 15, 220 (1978).

8) Nagao, T.: Trans. J.S.M.E., 44, 1912 (1978).

9) Suzuki, M., K. Makino, M. Yamada and K. Iinoya: Kagaku Kogaku Ronbunshu, 6, 59 (1980). 
10) Ouchiyama, N. and T. Tanaka: Ind. Eng. Chem. Fundam., 19, 340 (1980).

11) Tory, E.M., B.H. Church, M.K. Tam and M. Ratner: Can. J. Chem. Eng., 51, 484 (1973).

12) Nakagaki, M. and H. Sunada: Yakugaku Zasshi, 83, 73 (1963).

13) Shinohara, K., H. Kobayashi, K. Gotoh and T. Tanaka: J. Soc. Powder Technol., Japan, 2, 352 (1965).
14) Shinohara, K. and T. Tanaka: Kagaku Kogaku, 32, 88 (1968).

15) Bennett, H.: J. Appl. Phys., 43, 2727 (1972).

16) Bernal, J.D. and J. Mason: Nature, 188, 910 (1960). 